\title{
Early Failure Of External Fixation In The Management Of War Injuries
}

JC Clasper, SL Phillips

\author{
ABSTRACT \\ Objective \\ To review external fixation in the \\ management of war injuries.
}

\section{Method}

We prospectively followed up 15 external fixators (14 patients) applied in the management of war injuries. All these patients were treated at 202 Field Hospital during the 2003 Gulf Conflict.

\section{Results}

Of the 15 fixators, $13(86.7 \%)$ required early revision or removal due to complications of the injury or the fixator. Instability was a problem with 10 fixators (67\%), pin loosening was noted with 5 fixators $(33 \%)$ involving twelve pins, and a significant pin track infection developed at 14 pin sites (3 fixators $-20 \%$ ), which failed to resolve despite intravenous antibiotics.

\section{Conclusions}

This study demonstrates a very high early complication rate of external fixation in the management of military injuries and cautions against its universal acceptance. If used, consideration must be given to the optimum time of frame application, whether at the time of initial debridement or at a later operation, and the optimal frame design, which will depend on the specific bone and fracture pattern. Pin site care must also be considered, particularly with the restrictions imposed by the military environ-ment.

Introduction

Limb wounds are the most common injuries seen during armed conflict, accounting for as many as $70 \%$ of all wounds, with the lower limb most commonly involved. Many of these will be high-energy wounds, and half of the injuries may be associated with a fracture (1).

The principles of treatment for war wounds remain the same as any trauma, with the initial aim being to identify and treat any life-threatening injuries. The principles of wound care are also similar to the civilian environment, surgical debridement and adequate lavage, stabilisation of the limb, and the use of appropriate antibiotics.

The method of stabilisation of fractures has been a matter of debate for some years. Previously, more conservative methods such as plaster would have been the preferred option, however, war wounds are often associated with severe soft tissue injury, multifragmentary fractures, and multiple injuries, which makes plaster less than ideal. Unfortunately internal fixation with plates or the use of intramedullary nails has been reported to have a high complication rate, particularly infection $(2,3)$.

The use of an external fixator for managing war wounds has been debated, with opinions both for and against its use (4-10). At the end of the Second World War, Cleveland in a post war summary reported that it was associated with a very high complication rate, and the devices were removed from American military hospitals (4). More recent reports have also documented a high complication rate when external fixation has been used to treat military injuries $(6,7,11)$, but this must be balanced against the fact that many of these injuries are highly contaminated and associated with significant tissue injury. As such they would be expected to have a high complication rate anyway.

Despite its complications, external fixation has been widely accepted and in some reports has been used in the management of all ballistic fractures (12). External fixation has even been recommended on logistic rather than medical reasons (8). This prospective study reports a very high early complication rate of external fixation and cautions against its universal acceptance.

\section{Patients and Methods}

During the recent Gulf conflict, the authors were part of the orthopaedic department of 202 Field Hospital, which received the majority of patients who had external fixators 
applied by the British Armed Forces. This study reports our experience with the use of external fixation in the management of ballistic injuries. Not all of the fixators were applied at 202, in some cases external fixation was carried out at a forward surgical location as part of resuscitative surgery, or at another field hospital and subsequently transferred to our hospital. All frames were applied or directly supervised by consultant orthopaedic surgeons and all case notes were available for the patients who had external fixation performed prior to transfer to our hospital.

The majority of patients with ballistic injuries were Iraqi citizens, both military and civilian. As a result of difficulties in accessing an already overloaded Iraqi medical system, the majority of the patients remained as inpatients for several weeks. This allowed a prospective study of their follow-up to be carried out.

Two different types of fixator were applied as the Centrafix external fixator (Central Orthopaedics Ltd, High Wycombe, UK) is currently being replaced by the Hoffman II fixator (Stryker). Both devices are manufactured and package for military use, with one sterile package containing, 4 selfdrilling, self-tapping pins, $1 \mathrm{bar}$, as well as pin-to-bar connectors.

All pins were inserted by hand, as recommended by the manufacturers, using aseptic techniques in a dedicated operating theatre setup. The operating theatres, however, were in field hospitals, and in the majority of cases this was in tents without any appropriate air conditioning or sterile filters. Following application weight-bearing through the affected limb was restricted, and daily pin site care was carried out to remove any crusting and prevent fluid accumulation.

\section{Results}

A total of 17 patients (18 external fixators) were managed at our hospital. Two patients were excluded, as they had sustained blunt trauma, the remaining 15 patients (16 external fixators) had sustained penetrating ballistic injuries. Follow-up was available for 14 (15 external fixators); the remaining patient, with multiple injuries from blast, was transferred out of the hospital within 24

Table 1. Injuries treated by external fixator, their complications and outcome.

\begin{tabular}{|lcccccc|}
\hline Fixator & \multicolumn{3}{c}{ Complication } & \multicolumn{2}{c|}{ Outcome } \\
\hline $\begin{array}{c}\text { Total } \\
\text { Number }\end{array}$ & Instability & $\begin{array}{c}\text { Pin } \\
\text { Loosening }\end{array}$ & $\begin{array}{c}\text { Pin } \\
\text { Track } \\
\text { Infection }\end{array}$ & Removed & Revised \\
\hline $\begin{array}{l}\text { Lower } \\
\text { Limb }\end{array}$ & 11 & 8 & 4 & 3 & 6 & 5 \\
\hline $\begin{array}{l}\text { Upper } \\
\text { Limb }\end{array}$ & 4 & 2 & 1 & 0 & 1 & 1 \\
\hline Total & 15 & 10 & 5 & 3 & 7 & 6 \\
\hline
\end{tabular}

hours of external fixation of an open tibial fracture, and no further information was obtainable. Of the 15 frames, 5 had not been applied at the time of the initial surgery as it had been felt at the time of initial debridement that external fixation was not required. This decision was subsequently changed at the time of wound closure after 35 days to optimise soft tissue healing.

There were 12 males and 2 females with a mean age of 33 years (range 18-63). The details of the fixators are presented in Table 1 , one patient had 2 fixators applied, an external fixator placed across the wrist as well as one applied between the pelvis and forearm to stabilise a severe blast injury to the wrist, associated with a severe soft tissue injury treated by a groin flap.

All external fixators were applied by British military facilities, 2 as part of forward surgery, the remainder at Field Hospitals or the RFA Argus.

Of the 15 fixators, 13 (87\%) required early revision or removal due to complications of the injury or the fixator. Seven (2 Centrafix, 5 Hoffman frames) required early removal at a mean of 9.1 days (range 1-19) after application (10.1 days after original injury), 3 when the limb required amputation, 2 due to multiple infected pins, 1 due to multiple infected loose pins and 1 patient in whom it was felt that the external fixator was inadequate and that a different method of stabilisation was more appropriate. Four of the frames had been applied at the time of the initial debridement and 3 had been applied after 3 days ( 2 frames) and 5 days.

Six (5 Centrafix, 1 Hoffman frames) required early revision at a mean of 5.9 days (range 1-22) after application (all had been applied at the initial debridement), 5 were revised at the time of definitive wound closure due to instability at the fracture site and 1 bridging knee fixator was revised to a tibial fixator due to instability and loss of fracture reduction.

Following revision, follow up was possible for a further 21 days (range 5-35) without any further complications. Complications of individual fixators are noted in Table 1 .

Instability was a problem with 10 fixators $(67 \%)$; unfortunately all these fixators had initially been applied to stabilise the fracture site to optimise soft tissue recovery. Seven fixators were revised and 3 were removed. Revision was carried by removing loose pins, inserting additional pins and the placement of additional bars. Three frames were removed and the patients managed by different methods of stabilisation. A radial fracture was subsequently managed by plaster and one femoral fracture was managed by skeletal traction. With the third patient, as well as external fixation removal, above-knee amputation was required due to an unsalvageable vascular injury.

Pin loosening (13) was noted with 5 
fixators (33\%) involving twelve pins; 4 Centrafix pins (2 patients) were noted to have stage 3 loosening (considerable motion between pin and bone) after 1 day and 8 Hoffman pins ( 3 patients) were noted to be loose. Of the Hoffman pins stage 2 (slight motion noticeable between pin and bone) loosening was present with 2 pins, and stage 3 loosening was present with 6 pins, all of which were infected.

A significant pin track infection developed at 14 pin sites (3 fixators - 20\%), which failed to resolve despite intravenous antibiotics. All 3 fixators were removed after a mean of 15.5 days (range 14-19) and 2 patients were managed by skeletal traction. With the 3rd patient it was felt that

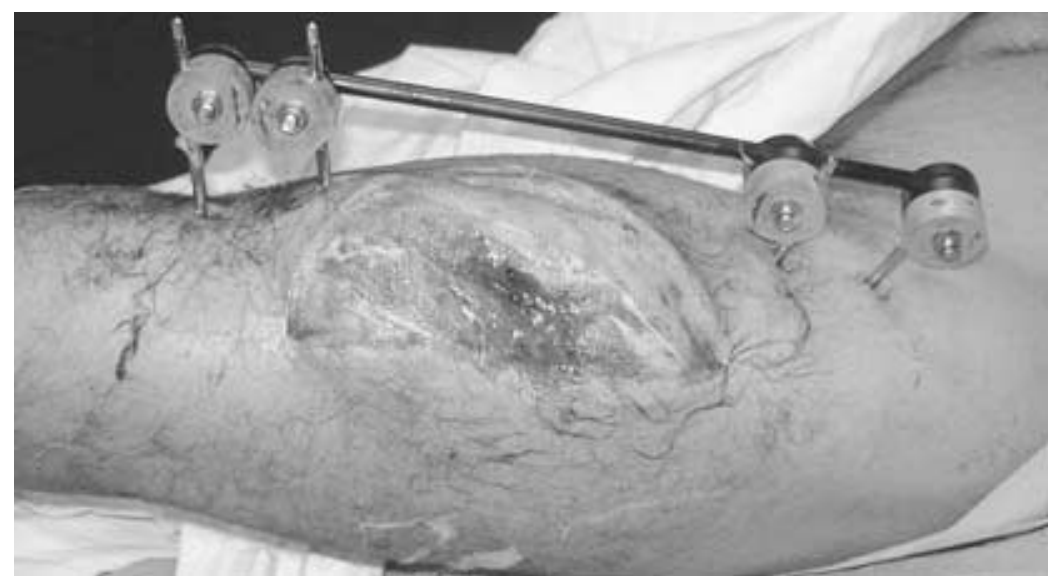

Fig 1a. Oedematous wound, with soft tissue swelling and ischaemia (Case 1).

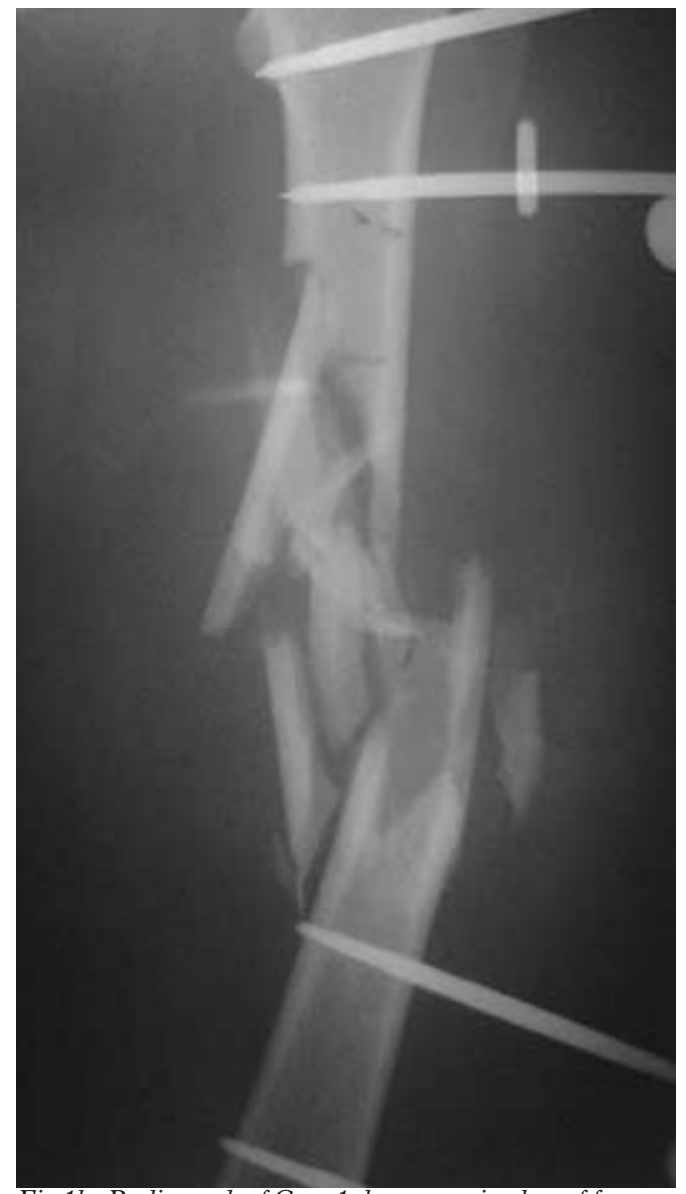

Fig 1b. Radiograph of Case 1 demonstrating loss of fracture reduction. stabilisation was unnecessary and the patient was mobilized.

Only 2 fixators did not require early removal or revision, both had multifragmentary injuries, one to the ulna and the other to the carpus; neither had been applied at the time of the original debridement, but had been applied at 3 and 5 days post injury. Follow-up was possible for 30 and 32 days, with no complications and new bone formation evident on radiographs. It was anticipated that external fixation would be continued for a total of 6-8 weeks.

\section{Illustrative Cases}

Case 1 - A 29 year old Iraqi soldier was admitted to a Field Hospital with a highenergy multifragmentary fracture of the femur. Initial treatment consisted of debridement and the application of a Centrafix fixator. After 5 days he was subsequently transferred to our hospital for further management. On admission the wound was oedematous, with soft tissue swelling and ischaemia (Figure 1a). Radiographs demonstrated loss of fracture reduction (Figure 1b) and at surgery, there was noted to be marked instability at the fracture site. Subsequent management consisted of further debridement, revision of the fixator and a later posterior thigh flap. Wound healing occurred although he was transferred out of our hospital before fracture healing occurred.

Case 2 - A 20 year old Iraqi soldier sustained a gunshot wound to the upper tibia. Initial treatment at a Field Hospital consisted of debridement and a Hoffman cross knee external fixator, with later skin graft. Early wound healing was uncomplicated, but check radiographs after 22 days demonstrated loss of reduction and an unacceptable position (Figure 2). At revision the single pin in the proximal tibia was loose and was replaced together with revision to a below knee frame. The patient was transferred out of our care a few days later and no further information is available.

Case 3 - A 34 year old Iraqi soldier sustained multiple gunshot wounds, requiring a laparotomy. In addition he sustained a multifragmentary fracture of the radius, treated by debridement and external fixation (Figure 3a) and was transferred to our hospital. On admission there was noted to be a single pin fixator that was not adding to stability, and revision was undertaken. At the time of revision there was noted to extensive damage to the flexor compartment together with the median nerve. In view of this it was felt that the intact ulna would provide sufficient stability and that external fixation would be of little benefit, therefore, the frame was removed rather than revised. Wound healing occurred following split-skin grafting (Figure 3b), although the patient was 


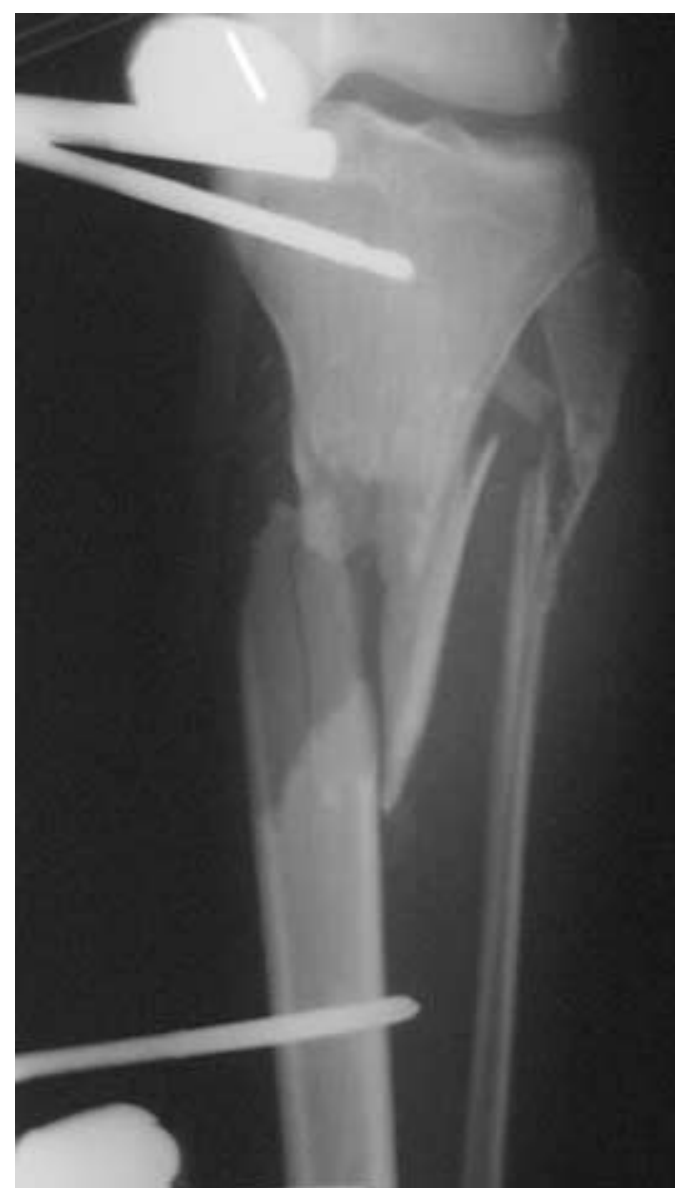

Fig 2. Radiograph of Case 2 demonstrating loss of reduction and an unacceptable position .

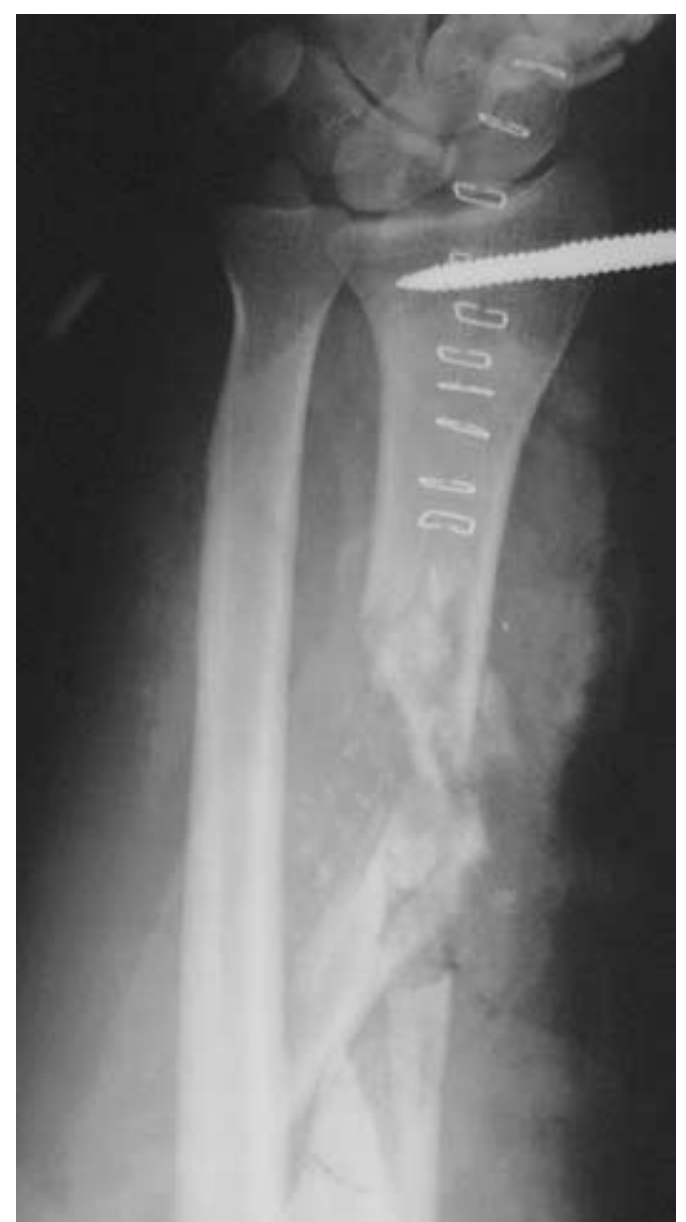

Fig 3a. Case 3 with inadequate fixation and lack of stability. transferred out of our hospital after 28 days, prior to fracture healing.

\section{Discussion}

The use of external fixation in the management of war injuries has been controversial, particularly since the Second World War. One of the difficulties in identifying its role is the lack of adequate follow-up, due to the rapid evacuation of patients (14). The main exception to this is the International Committee of the Red Cross (ICRC) who do not evacuate their patients, and their recommendation is that external fixation is occasionally indicated, but that it should not be carried out at the time of initial surgery (5). This is in contrast to other authors who have used external fixation for the majority of fractures (12). The advice of the ICRC must be considered in light of the high early failure rate reported in this study. It is possible that a high failure rate would have occurred in the other studies if they had been able to follow-up their patients. Although our follow-up is relatively short, this is not a weakness of this study as the majority of frames had failed by this stage anyway.

Instability occurred in $67 \%$, and as the principal indication for external fixation was to provide bony stability, this is unacceptably high. We defined instability as when gross motion was present at the fracture site such that soft tissue healing could be compromised, or delayed fracture healing anticipated.

The cause was multifactorial, but was related to injury severity and frame design. In the majority of cases the basic frame design conformed to previous military medical recommendations of 2 pins on either side of the fracture with one connecting bar. This had been considered adequate, balancing the need for stability against the limitations placed on equipment and operating time that are imposed when operating in the military environment, particularly near a front line.

With the multifragmentary nature of the fractures and the resulting gap after debridement, the recommended design of 2 pins either side, with a single bar was inadequate. In addition due to the design of the Hoffman pin clamp, the 2 pins are close together, which also reduces stability. Although it was intended that only one military pack would be used per injury, multiple packs were commonly used at the time of revision to allow 3 pins either side of a fracture or multiple bars to be used. This obviously has logistic implications for the military. The high failure rate of the frames has meant that the previous guidelines must be questioned, either delaying the application of the frame, or accepting the need for more equipment and longer operating times.

For all long bone fractures at least 2 bars were required, with at least 3 with the femur, 


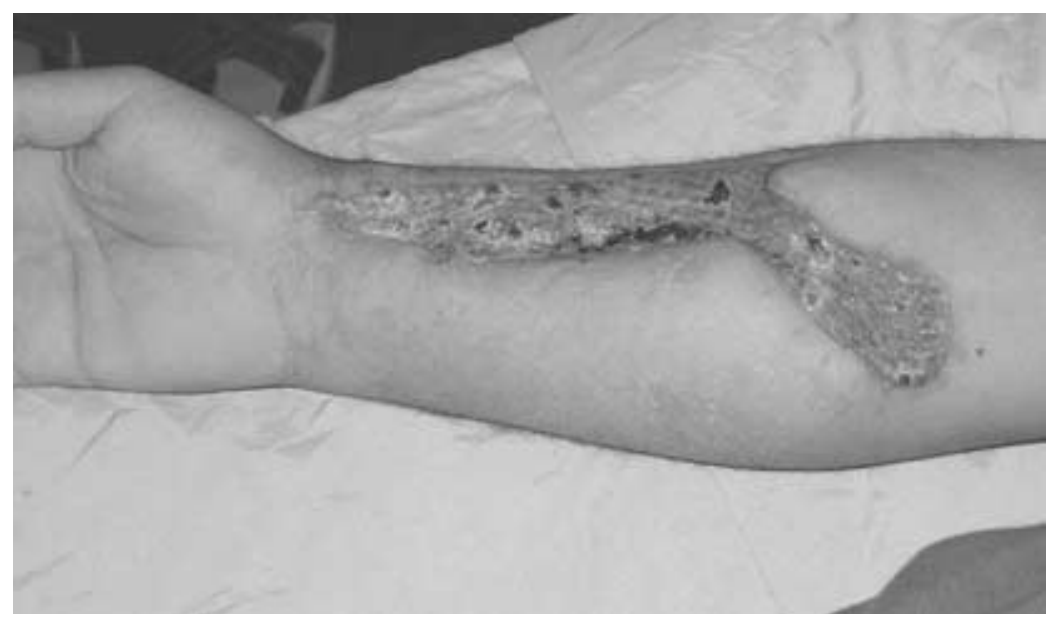

Fig 3b. Case 3 demonstrating wound healing following split-skin grafting with stabilisation by plaster.

and we believe this more rigid frame design reduced the incidence of pin loosening, infection or loss of reduction in the short term following revision. Even at the time of revision, with a more rigid frame design, instability was still a problem and acute limb shortening was carried out to increase stability in 4 of the 6 patients who underwent revision.

In addition bridging fixators across the knee for distal femoral or proximal tibial fractures proved very unstable and we would not recommend this method of stabilisation for ballistic injuries using the current military equipment. Femoral fixators were also associated with instability, pin loosening and infection, and as we and other authors have successfully treated ballistic femoral fractures in skeletal traction we would recommend that this is considered as an alternative method of stabilisation to external fixation, particularly at the initial operation. Although there will still be instability at the fracture site, it would appear that the generation and accumulation of fluid around a fixator pin is the main factor in subsequent infection, rather than movement at the fracture site.

For those fractures where external fixation is the method of choice then we would suggest that the frame is constructed to achieve maximum stability, using additional pin to bar clamps. This allows wide separation of the pins on either side of the fractures, and is more stable than using a single clamp with the pins close together. Instability is also a major factor in the pathogenesis of pin track infection and further emphasises the need for bony stability.

Pin tract infection rates were high and this has also been previously reported in the military environment (11). This has been postulated as a potential problem and may be related to the extensive contamination that occurs with ballistic injuries (15). A further factor in the high infection rate of military wounds is likely to be the delay in initiating surgical care, which in our patients was approximately 6 hours for allied casualties and 28 hours for Iraqi casualties. Pin tract infection is a major concern and will lead to chronic problems as well as compromising secondary methods of stabilisation (6).

In addition although the fixators were applied using sterile techniques this was carried out in tents with no means of reducing any bacterial contamination in the air. As the initial debridement has to be carried out in this environment, this also supports the recommendation that external fixators are not applied at the time of initial surgery, but at a later stage in a more sterile environment. Pin care is also relevant and daily pin care was carried out at $202 \mathrm{fd}$ hospital. It is impossible to be sure what was done prior to evacuation to us, although most patients were evacuated soon after the initial application. This is a major concern in the military situation as frequent and prolonged evacuation of casualties may be required, which would prevent regular pin care. This may be another reason to delay application until a base hospital when pin insertion and after care can be carried out in a more controlled, sterile environment. Delayed application had been carried out with 5 patients, but all of these patients were evacuated to our hospital soon after the frame was applied compromising both wound and pin care. It may be appropriate to not only delay the application of the frame, but also to delay further evacuation of the patient after a fixator has been applied, to ensure appropriate wound and pin care. However, this would have operational implications to the military, where rapid evacuation down the medical chain is the norm.

Pin loosening was also a major problem, and seemed to be related to 2 factors, pin type and pin track infection. With the Centrafix pins, difficulty in inserting the selfdrilling pins has been noted previously by military orthopaedic surgeons and was one of the reasons for its replacement. When a pin was difficult to insert, it was universally noted to be loose, and this is likely to be due to bony damage, particularly the threads. All 4 Centrafix pins found to be loose at 24 hours had been documented to be difficult to insert. We would, therefore, agree with the decision to remove this external fixator from British Military medical units.

All Hoffman pins that were loose were infected, but it is impossible to say which complication occurred first. Loose pins are more likely to become infected, but infected pins are also more likely to loosen.

With the high early complication rate, the early application of the external fixator appears to have been of limited benefit in the context of military injuries. This is a major concern, particularly as previous authors have recommended external fixation on the basis that it is effectively risk-free (9). Given this, delayed application of the frame, until 
definitive closure of the wound, and continual observation of the patient, may have been more appropriate, and would have reduced the initial operative time, and possibly morbidity. This is the recommendation of the ICRC (5) and we would support this opinion. This must, however, be balanced with the possibility that delaying external fixation may in itself increase the infection risk due to the lack of stability. Other methods of stabilisation, including skeletal traction should be considered, and we did successfully treat some patients with this method.

Amputation was required for 4 limbs. Although this may not appear to be true failure of external fixation, amputation was required in 2 patients for overwhelming infection, which occurred in association with instability at the fracture site in both patients, and a severe pin track infection in one. In addition a third amputation was carried out due to a failed vascular repair, again in association with an unstable frame; failure of external fixation may have been a factor in the loss of these 3 limbs.

\section{Conclusion}

We have demonstrated a high early failure rate with the use of external fixation for ballistic injuries in the military setting, and would caution against its universal acceptance, particularly in forward surgical locations. For many fractures plaster or skeletal traction provide an alternative option. When external fixation is required, multiple pins and bars must be utilised, bridging frames should be avoided, and if necessary acute limb shortening considered. Even with this there is likely to be a high complication rate due to pin track infection and loosening, and amputation must still be considered as a possible outcome.

\section{References}

1. Islinger RB, Kuklo TR, McHale KA. A review of orthopaedic injuries in three recent US military conflicts. Mil Med 2000;165:463-465.

2. Furlong R, Clark MP. Missile wounds involving bone. Br J Surg 1948;War Supplement No. II:291310.

3. Rich NM, Metz CW, Hutton JE, Baugh JH, Hughes CW. Internal versus external fixation of fractures with concomitant vascular injuries in Vietnam. $\mathcal{F}$ Trauma 1971;11:463-473.

4. Clevland $M$. The management of compound fractures -Techniques of fracture management. In: Surgery in World War II, European theatre. Washington: Dept. of Army, 1956, 109-118.

5. Coupland RM. War wounds of bone and external fixation. Injury 1994;25:211-217.

6. Has, Jovanovic S, Wertheimer B, Mikolasevic I, Grdic P. External fixation as a primary and definitive treatment of open limb fractures. Injury 1995;26:245-248.

7. Labeeu F, Pasuch M, Toussaint P, Van Erps S. External fixation in war traumatology: Report from the Rwandese war. F Trauma 1996;40:S223-S227.

8. McHenry T, Simmons S, Alitz C, Holcomb J. Forward surgical stabilization of penetrating lower extremity fractures: circular casting versus external fixation. Mil Med 2001;166:791-795.

9. Rowley DI. The management of war wounds involving bone. F Bone foint Surg[Br] 1996;78B:706-709.

10. Zinman C, Reis ND. External fixation in wartime limb surgery. Israel f Med Sci 1984;20:308-310.

11. Dubravko H, Zarko R, Tomislav T, Dragutin K and Vjenceslav N. External fixation in war trauma management of the extremities - Experience from the war in Croatia. F Trauma 1994;37:831-834

12. Maričevic A, Erceg $M$. War injuries to the extremities. Mil Med 1997;162:808-811.

13. Burny F. The pin as a percutaneous implant. Orthopaedics 1984;7:610-615.

14. Spalding TJW, Stewart MPM, Tulloch DN, Stephens KM. Penetrating missile injuries in the GulfWar 1991. Br F Surg 1991;78:1102-1104.

15. Clasper JC. Secondary intramedullary nailing of the tibia in an animal model of an external fixator pin track infection. Thesis for Doctor of Medicine, University of Southampton; August 2001:78. 